\title{
Control of soliton collision-induced enhancement of supercontinuum bandwidth in photonic crystal fiber by variation of pump pulse duration
}

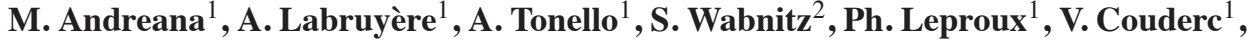

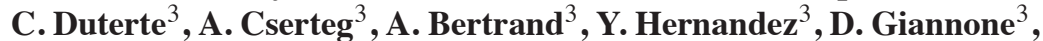 \\ S. Hilaire ${ }^{4}$, G. Huss ${ }^{4}$ \\ ${ }^{1}$ XLIM, Université de Limoges, 123 avenue Albert Thomas, 87060 Limoges cedex, France \\ ${ }^{2}$ Dipartimento di Ingegneria dell'Informazione, Universitá di Brescia, via Branze 38, 25123 Brescia, Italy \\ ${ }^{3}$ Multitel asbl, Parc Initialis, 2 rue Pierre et Marie Curie, 7000 Mons, Belgique \\ ${ }^{4}$ Leukos, Ester Technopole, 1 avenue d'Ester, 87069 Limoges cedex, France \\ marco.andreana@xlim.fr
}

\begin{abstract}
We investigate experimentally and theoretically the impact of input pulse width varying from 500 fs to $10 \mathrm{ps}$ on supercontinuum generation. We show that the spectral broadening is dramatically extended for the longer input pulses.
\end{abstract}

(C) 2011 Optical Society of America

OCIS codes: $190.4370,190.5530,260.2160,320.6629$.

Supercontinuum (SC) generation is a nonlinear phenomenon in which an intense light pulse is dramatically converted into a broadband spectrum of light when propagating in an optical fiber. Over the last decade, this effect has attracted much attention because of applications in many diverse fields, notably in laser-frequency metrology, biology or medicine [1]. Since the early experiences of SC generation using femtosecond pump lasers [2], alternative regimes of inputs have been investigated to cover a full range of possible durations including picosecond, nanosecond and continuous-wave $(\mathrm{CW})$ laser sources which may potentially benefit from more compactness and low costs [3-5]. But these different pulse width are usually associated with qualitatively different nonlinear mechanisms. In the femtosecond regime, the spectral broadening is typically driven by soliton fission, whereas for longer pump pulses or CW, SC generation is initiated by modulational instability (MI). Whereas both regimes are well understood individually, there has been no investigation on the transient regime of input pulse duration. In this paper, we provide an experimental

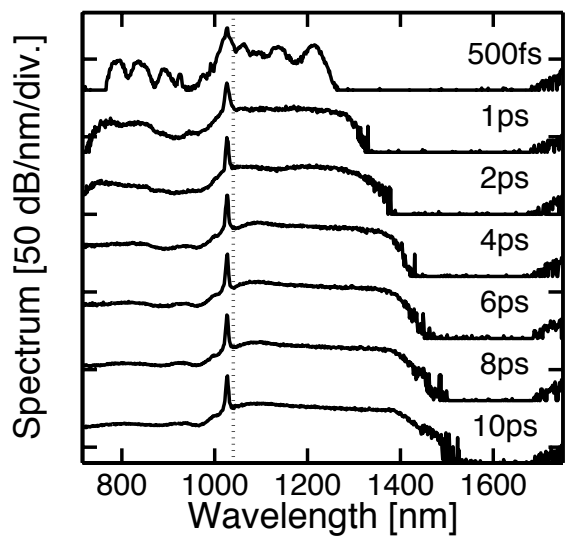

Fig. 1. Experimental output SC spectra for different input pulse durations and constant input peak power of $3 \mathrm{~kW}$ after propagation in $8 \mathrm{~m}$ of photonic crystal fiber. The dashed line represents the zero dispersion wavelength.

comparison between SC spectra obtained when varying the input pulse width from the femtosecond to the picosecond time scale. This is rendered possible by the use of a pulsed laser with continuously tunable duration from 500 
fs to $10 \mathrm{ps}$ while keeping the peak power at the constant value of $3 \mathrm{~kW}$. We use $8 \mathrm{~m}$ long of photonic crystal fiber characterised by a $5 \mu \mathrm{m}$ core diameter, a $3 \mu \mathrm{m}$ hole-to-hole pitch and an air filling fraction of 0.5 . Its zero dispersion point is located at $1040 \mathrm{~nm}$. The most remarkable result of our study is that the infrared (IR) spectral broadening is significantly extended for the longer input pulses. Figure 1 shows the experimental spectra obtained for the different pulse durations showing evidence that SC is dramatically extended towards infrared at the longer pulse width. Through numerical simulations based on the nonlinear Schrödinger equation we were able to find a supporting interpretation for this peculiar feature. The resulting simulation spectra are depicted in Fig. 2 where the same tendancy is numerically reproduced. Whenever an intense optical pulse is injected in an optical fiber in the anomalous dispersion regime, the lowest threshold nonlinear effect is MI. Subsequently MI leads to the break-up of the pump pulse into a periodic train of solitons. Afterward Raman effect acts as a perturbation that breaks the symmetry of the soliton train and hence most of the energy in the IR spectrum takes the form of individual solitons shifting towards lower frequencies via the Raman-induced self-frequency shift (SSFS). Since the SSFS is proportional to the square of the soliton power, a soliton which is initially generated in the central part of the input pulse down-shifts faster than the neighbouring solitons. Because of the corresponding dispersion-induced slowing down, this central soliton collides with all subsequent solitons in the train [6]. Another important consequence of Raman gain is that during each collision the slower, lower-frequency central soliton acquires energy from higher-frequency solitons while reducing its time width down to the femtosecond range so as to maintain the shape of a fundamental soliton [7]. This particular soliton which can be dramatically amplified is often named as "rogue" soliton and for convenience we use here this terminology. As a result of all collisions, rogue solitons run-away from the initial soliton train and a broad SC is thus generated. Indeed, it has recently been pointed out that the large soliton frequency down-shifts that are observed in the process of SC generation in optical fibers cannot be explained in terms of the SSFS alone. In fact, the collision-enhancement of the rogue soliton down-shift rate is crucial for understanding the mechanism of SC generation in optical fibers. In this work we derive a

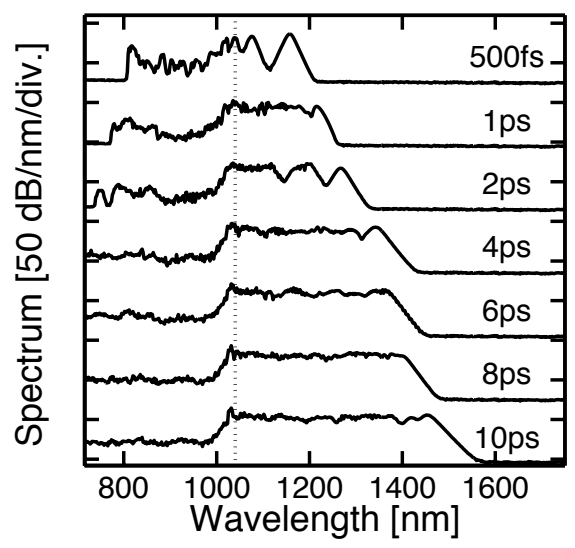

Fig. 2. Calculated SC spectra from numerical simulations for different input pulse durations and constant input peak power of $140 \mathrm{~W}$ after a propagation distance of $10 \mathrm{~m}$. The dashed line represents the zero dispersion wavelength.

simple analytical estimate for the collision-induced enhancement of the SSFS. We point out that the frequency-down shift rate of the rogue soliton that emerges from the collisions grows larger with the number of collisions, hence with the time duration of the input pump pulse. Therefore the bandwidth of the generated SC may be substantially enlarged by increasing the input pulse width. Experiments carried out from the 500 fs to $10 \mathrm{ps}$ pump duration regimes provide a good confirmation of this prediction.

Additionnally, we show that the above mentionned mechanism of amplification of the rogue soliton eventually stops in three situations, respectively: (i) when the rogue soliton temporally walks off from the rest of the infrared solitonic components (ii) when the frequency detuning between colliding solitons becomes larger than the Raman gain bandwidth or (iii) when the colliding soliton has been fully depleted by the rogue soliton. Our estimate for the SSFS accounts for these stopping mechanisms and hence saturation of the SSFS rate providing satisfying agreement with the experiments that we have carried out here. Figure 3 shows spectral trajectories of each individual infrared soliton obtained from our estimate for an input pulse duration of $2 \mathrm{ps}$ and peak power of $140 \mathrm{~W}$. The input pulse initially splits into a train of seven individual solitons. The brown line indicates the position of the rogue soliton which is considerably 
more frequency down-shifted. Note that eventually some of the solitons are fully depleted during propagation and the corresponding lines vanish in Fig. 3. It is important to notice that the predicted SSFS has the meaning of an average

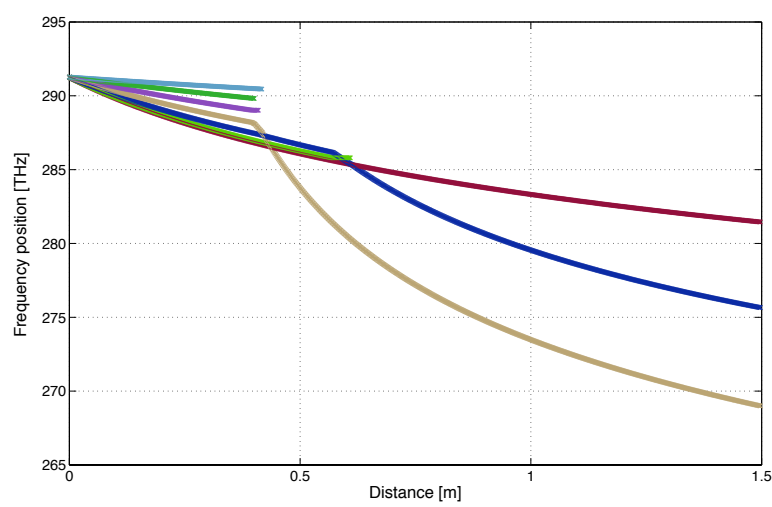

Fig. 3. Spectral position as a function of propagation distance for each individual infrared soliton obtained from our estimate for an input pulse duration of $2 \mathrm{ps}$ and peak power of $140 \mathrm{~W}$. The brown line indicates the position of the rogue soliton.

value when compared with the numerical simulations including a random noise seed, or the experiments. In fact, it is well-known that the observed SC spectra represent an ensemble average of all spectra resulting from the various femtosecond rogue solitons that are obtained with different pump pulses. Indeed, in obtaining our estimate for the SSFS we neglected rogue soliton power variations owing to the interplay between nonlinearity and noise such as: (i) the relative phase dependence of the first collision involving two almost frequency-degenerate solitons; (ii) additional SSFS-induced collisions between different solitons composing the MI-generated train; (iii) soliton dressing effects and small dispersive radiations emitted by solitons while propagating or colliding; (iv) collisions involving three or more solitons, etc.

In this paper, we have performed experiments of SC generation in a photonic crystal fiber by varying the input pulse width of the pump laser. We found out that infrared spectral broadening is strongly enhanced when increasing the input pulse duration from 500 fs to $10 \mathrm{ps}$. We emphasize the fact that the mechanism of infrared spectral broadening can be essentially described by the collisions between solitons and amplification of a rogue soliton.

\section{References}

1. J. M. Dudley, G. Genty, and S. Coen, “Supercontinuum generation in photonic crystal fiber," Rev. Mod. Phys. 78, 11351184 (2006).

2. J. K. Ranka, R. S. Windeler, and A. J. Stentz, "Visible continuum generation in air-silica microstructure optical fibers with anomalous dispersion at $800 \mathrm{~nm}$," Opt. Lett. 25, 2527 (2000).

3. S. Coen, A. H. L. Chau, R. Leonhardt, J. D. Harvey, J. C. Knight, W. J. Wadsworth, and P. St. J. Russell, "Whitelight supercontinuum generation with 60-ps pump pulses in a photonic crystal fiber," Opt. Lett. 26, 13561358 (2001).

4. E. Räikkönen, G. Genty, O. Kimmelma, M. Kaivola, K. P. Hansen, and S. C. Buchter, "Supercontinuum generation by nanosecond dual-wavelength pumping in microstructured optical fibers," Opt Express 14, 7914-23 (2006).

5. A. Mussot and A. Kudlinski, "19.5 W CW-pumped supercontinuum source from 0.65 to $1.38 \mu \mathrm{m}$," Electron. Lett. 45, 2930 (2009).

6. M. N. Islam, G. Sucha, I. Bar-Joseph, M. Wegener, J. P. Gordon, and D. S. Chemla, "'Femtosecond distributed soliton spectrum in fibers," J. Opt. Soc. Am. B, 6, 1149-1158 (1989).

7. M.H. Frosz, O. Bang, A. Bjarklev, "Soliton collision and Raman gain regimes in continuous-wave pumped supercontinuum generation,” Opt. Express 14,9391-9407 (2006). 